\title{
Up-regulation of liver Pcsk9 gene expression as a possible cause of hypercholesterolemia in experimental chronic renal failure
}

\author{
Elzbieta Sucajtys-Szulc $^{1} \cdot$ Marek Szolkiewicz $^{1} \cdot$ Julian Swierczynski $^{2}$. \\ Boleslaw Rutkowski ${ }^{1}$
}

Received: 1 July 2015/Accepted: 8 October 2015/Published online: 19 October 2015

(c) The Author(s) 2015. This article is published with open access at Springerlink.com

\begin{abstract}
Dyslipidemia commonly present in patients with chronic kidney disease (CKD) has been recently linked to increased proprotein convertase subtilisin/kexin type 9 (PCSK9) serum concentration. We tested a hypothesis that increased liver PCSK9 biosynthesis could be partially responsible for the elevated circulating PCSK9 level, and subsequently contribute to hypercholesterolemia observed in subjects with CKD. Rat model of chronic renal failure (CRF) was used in the study. Animals underwent a $5 / 6$ nephrectomy or a sham operation. Liver expression of Pcsk9, sterol regulatory element-binding transcription factor 2 (Srebf-2), and $\beta$-actin were quantified by real-time RT-PCR. Liver protein levels of PCSK9, LDL-receptor (LDL-R), and SREBF-2 were analyzed using Western blotting. Serum PCSK9 concentration was estimated by immunoassay. Rats with an experimental CRF as compared to pair-fed and control ones were characterized by: (a) an up-regulation of liver Pcsk9 and Srebf-2 genes expression with parallel increase of serum PCSK9 concentration; (b) a decrease in liver LDL-R protein level, and (c) an increase of serum total and LDL-cholesterol concentrations. We also found significant correlations between serum creatinine and liver PCSK9 mRNA levels $(r=0.88, p<0.001)$ and between serum creatinine and circulating PCSK9 levels $(r=0.73$, $p<0.001)$. The results suggest that a rat model of CRF is associated with an increased liver Pcsk9 gene expression.
\end{abstract}

Boleslaw Rutkowski

bolo@gumed.edu.pl

1 Department of Nephrology, Transplantology and Internal Medicine, Medical University of Gdansk, ul. Debinki 7, 80-211 Gdańsk, Poland

2 Department of Biochemistry, Medical University of Gdansk, ul. Debinki 1, 80-211 Gdańsk, Poland
The coordinated up-regulation of Pcsk9 and Srebf-2 genes expression suggests that SREBF-2 may play a key role in regulation of Pcsk9 gene expression, circulating PCSK9 level, and hypercholesterolemia in experimental CRF.

Keywords PCSK9 - SREBF-2 - LDL - LDL-R . Hypercholesterolemia $\cdot$ CRF

\section{Introduction}

Dyslipidemia, one of the most potent cardiovascular risk factor in patients with chronic kidney disease (CKD), significantly contributes to increased morbidity and mortality in this group of patients as compared to general population [1]. Moreover, lipid abnormalities themselves accelerate progression of CKD [2]. Therefore, an evaluation of the molecular mechanism leading to CKD-related lipid disturbances is necessary for a better understanding and treatment of the disease. In our prior studies, we have shown that hypercholesterolemia observed in experimental CRF is partially caused by the enhanced cholesterol biosynthesis associated with the up-regulation of Srebf-2 and $\mathrm{Hmg}-\mathrm{CoA}$ reductase genes expression as well as by a decrease in LDL-receptor (LDL-R) mRNA level [3-5]. However, in our in vivo experimental model, cholesterol biosynthesis rate was increased only twofold, while circulating cholesterol concentration was 3-4-times higher in CRF rats when compared to control [5]. Thus, one can assume that the increased cholesterol biosynthesis is not the only process contributing to hypercholesterolemia in experimental CRF rats.

Several papers published in last decade suggest that proprotein convertase subtilisin/kexin type 9 (commonly known as PCSK9; originally named NARC-1-neural apoptosis- 
regulated convertase-1) plays a crucial role in LDL-cholesterol (LDL-Ch) metabolism [6-9]. PCSK9 is synthesized mainly in hepatocytes (and in lesser amounts in intestine and other organs) [10] as a $74 \mathrm{kDa}$ soluble zymogen (proPCSK9) that undergoes autocatalytic cleavage into propeptide $(14 \mathrm{kDa})$ and PCSK9 $(60 \mathrm{kDa})$ in the endoplasmic reticulum [10]. Cleavage is necessary to activate the convertase and to allow its secretion into circulation [10]. PCSK9 secreted into the bloodstream plays a crucial role in the regulation of circulating LDL-Ch concentration by binding to hepatocytes LDL-R, promoting their intracellular degradation, and preventing LDL-R recycling to the cell surface [7, 9, 11, 12]. In liver, Pcsk 9 gene expression is up-regulated by SREBF-2 and down-regulated by cholesterol [11, 12]. PCSK9 null mice display increased LDL-R level and low circulating cholesterol concentration, whereas mice overexpressing PCSK9 exhibit high circulating cholesterol concentration [13, 14]. In humans, circulating PCSK9 level correlated positively with serum total and LDL-Ch concentration [15] and there are reports suggesting that elevated serum concentration of PCSK9 may play a role in early pathogenesis of atherosclerosis [16]. Thus, measuring circulating PCSK9 level and treating patients with human monoclonal PCSK9 antibodies [17] may have clinical significance. Recently, it has been shown that patients with nephrotic syndrome or patients with advanced renal failure treated by peritoneal dialysis displayed elevated plasma PCSK9 level [18]. Furthermore, in rats with experimental nephrotic syndrome, an up-regulation of liver PCSK9 was observed [19]. We also have recently reported that circulating PCSK9 concentration is significantly elevated in CKD patients [20] and we have suggested that PCSK9 overproduction could partially contribute to its elevated serum concentration found in patients with this disease [20]. We based this assumption on our previously reported data indicating that in rat model of CRF a liver Srebf-2 gene is overexpressed [5] and that $P c s k 9$ gene expression is up-regulated by SREBF-2 [21, 22]. The aim of the current study was to test the hypothesis that in rats with experimental CRF, liver Pcsk 9 gene expression is up-regulated and that SREBP-2 could be involved in this process. Our findings, presented here for the first time, provide new information about coordinated up-regulation of liver Pcsk9 and Srebf-2 genes expression leading to hypercholesterolemia observed in experimental CRF.

\section{Materials and methods}

\section{Animals}

Male Wistar rats (10 weeks old, weight $\sim 250 \mathrm{~g}$ at the beginning of the study) were used in all experiments. There were 10 animals per each studied group (CRF, pair-fed, control). Animals were kept in individual cages with free access to tap water, and with controlled lighting schedule (illuminated from 7 a.m to 7 p.m.). Experimental CRF was induced by two-stage (5/6) subtotal nephrectomy as described previously [23]. Average daily food intake was measured by the difference in weight between the amount of food provided and the amount remaining over a 1-day period. Pair-fed (sham-operated) rats received the amount of food (its composition has been described previously [24]) corresponding to what had been consumed by the matched CRF animals.

The non-fasted overnight rats were terminated (between 8 a.m. to 10 a.m.) 6 weeks after induction of renal failure. Liver was collected, and immediately frozen in liquid nitrogen for subsequent analyses of genes expression. The tissue was stored at $-80{ }^{\circ} \mathrm{C}$ until the analysis. Blood samples were collected from abdominal aorta, centrifuged, and the serum was used for determination of total protein, albumin, urea (BUN), creatinine, total cholesterol (TCh), and LDL-cholesterol (LDL-Ch). All animal procedures were conducted in agreement with our institutional guidelines for the care and use of laboratory animals.

\section{RNA isolation}

Total cellular RNA was extracted from frozen tissue by a guanidinium isothiocyanate-phenol/chloroform method [25]. The RNA concentration was determined from the absorbance at $260 \mathrm{~nm}$ and all samples had 260/280 nm absorbance ratio of about 2.0.

\section{cDNA synthesis}

First strand cDNA was synthesized from $2 \mu \mathrm{g}$ of total RNA (RevertAid $^{\mathrm{TM}}$ First Strand cDNA Synthesis Kit-Fermentas UAB, Lithuania). Prior to amplification of cDNA, each RNA sample was treated with RNase-free DNase I (Fermentas UAB, Lithuania) at $37^{\circ} \mathrm{C}$ for $30 \mathrm{~min}$.

\section{Determination of mRNA levels by real-time RT- PCR}

PCSK9, SREBF-2, and $\beta$-actin mRNA levels were quantified by real-time RT-PCR using Chromo4 Real-Time Detection System (Bio-Rad Laboratories, Inc; USA). Primers were designed with Sequence Analysis software package (Informagen, Newington, USA) from gene sequence obtained from Ensembl Genome Browser. The sequences of primer pairs (sense and antisense) used in this study are presented in Table 1. Real-time PCR amplification was performed in a $20 \mu \mathrm{l}$ volume using iQ SYBR Green Supermix (Bio-Rad Laboratories, Hercules, CA). Each reaction contained cDNA and $0.3 \mu \mathrm{M}$ of each primer. 
Table 1 Oligonucleotide primers sequences used in this study

\begin{tabular}{lll}
\hline Gene & Primer sequence & Accession Nr \\
\hline Pcsk9 & F: 5'-TGGCTGCATGACATTGCTTCTC-3' & 199253 \\
& R: 5'-GCACTGGAGAACCACACAGG-3' & \\
Srebf-2 & F: 5'-ACTGTCACTGGAGTCAGGTT-3' & 001033694 \\
& R: 5'-GACCAACAGCTTCACGAAGA-3' & \\
$\beta$-actin & F: 5'-TGTCACCAACTGGGACGATA-3 & 031144.3 \\
\hline
\end{tabular}

Control reactions, with omission of the RT step or with no template cDNA added, were performed with each assay. All samples were run in triplicate. To compensate for variations in the amount of added RNA, and in the efficiency of the reverse transcription, $\beta$-actin mRNA was quantified in corresponding samples as a control and the results were normalized to these values. Relative quantities of transcripts were calculated using the $2^{-\Delta \Delta C_{\mathrm{T}}}$ formula [26]. The results are expressed in arbitrary units (a. u.), with one unit being the mean mRNA level determined in the control group. Amplification of specific transcripts was further confirmed by obtaining the melting curve profiles and subjecting the amplification products to agarose gel electrophoresis.

\section{Serum PCSK9 concentration}

Serum PCSK9 concentration was estimated by immunoassay method using Rat Protein Convertase subtilisin/kexin type 9 (PCSK9) ELISA kit CSB-EL017647RA from CUSABIO BIOTECH CO.

\section{Western blot analysis of PCSK9, LDL-R, SREBP-2}

Frozen liver samples were homogenized in buffer containing 10 mM Tris- $\mathrm{HCl}$ (pH 6.8), 2 \% SDS, 10 mM DTT, proteinase inhibitors (Sigma), and then centrifuged at $15,000 \times g$ for $20 \mathrm{~min}$ at $20{ }^{\circ} \mathrm{C}$. Supernatants were collected, and the protein concentration was determined by Bradford assay. Supernatants, containing $20 \mu \mathrm{g}$ of total protein, were separated by $10 \%$ SDS-PAGE and electroblotted onto Immobilon ${ }^{\circledR}$ Transfer Membrane (Millipore). The following antibodies were used: mouse/rat Proprotein Convertase 9 polyclonal antibody (AF3985, R@D SYSTEMS), polyclonal antibody against LDL-R (sc11826, Santa Cruz Biotechnology), polyclonal antibody against SREBP-2 (sc-5603, Santa Cruz Biotechnology), and polyclonal rabbit antibody against Actin (A5060, Sigma). HRP-conjugated secondary antibody (sc-2030 and sc-2004) was obtained from Santa Cruz Biotechnology and HAF019 from R@D SYSTEMS. Immunodetection was accomplished with enhanced chemiluminescence by using Western Blotting Luminol Reagent (sc-2048, Santa Cruz Biotechnology). Band intensities of analysed proteins were quantified and normalized to the intensity of actin band, with CON value assumed as 1. Quantification of Western blots were performed using Quantity One software program (Bio-Rad Laboratories, Hercules, CA). The results are expressed in arbitrary units (a. u.), with one unit being the mean of corresponding protein (LDL-R and SREBP-2) level determined in the control group.

\section{Statistical analysis}

The statistical significance of differences between groups was assessed by one-way analysis of variance (ANOVA) followed by Student-Newman-Keuls and by one-way analysis of variance (ANOVA) followed by Tukey's post hoc test. The Sigma Stat software (Sigma Stat Inc.) was used. Differences between the groups were considered significant when $p<0.05$. All data are presented as means of values \pm standard deviation (SD).

\section{Results}

Serum concentration of creatinine increased approximately fourfold in CRF rats as compared to control (2.4 \pm 0.7 vs. $0.5 \pm 0.1 \mathrm{mg} / \mathrm{dl} ; p<0.001)$ or pair-fed $(2.4 \pm 0.7 \mathrm{vs}$. $0.6 \pm 0.1 \mathrm{mg} / \mathrm{dl} ; \quad p<0.001)$ animals, while in control $(\mathrm{CON})$ and pair-fed $(\mathrm{PF})$ ones they were essentially similar (not shown). Similarly, serum concentration of nitrogen urea increased approximately threefold in CRF rats as compared to $\mathrm{CON}(47.7 \pm 7.7$ vs. $15.1 \pm 1.2 \mathrm{mg} / \mathrm{dl}$; $p<0.001)$ or $\mathrm{PF} \quad(47.7 \pm 7.7$ vs. $14.9 \pm 0.8 \mathrm{mg} / \mathrm{dl}$; $p<0.001$ ) ones (not shown). Simultaneously, serum concentrations of $\mathrm{TCh}$ and $\mathrm{LDL}-\mathrm{Ch}$ in $\mathrm{CRF}$ rats were approximately threefold higher than in CON and PF ones (Fig. 1a, b). Presented data indicate that CRF induced by 5/6 nephrectomy corresponded to stage $5 \mathrm{CKD}$ in patients. Positive correlations between serum creatinine and TCh concentrations $(r=0.82 ; p<0.001)$ and between serum creatinine and LDL-Ch concentrations $(r=0.84$; $p<0.001$ ) were observed (not shown). This suggests that serum TCh and LDL-Ch levels are tightly related to progression of kidney disease.

Figure 1c shows the relative PCSK9 mRNA levels in liver of CON, PF, and CRF rats. The greatest level of liver 
Fig. 1 Liver Pcsk9 gene expression and serum cholesterol and PCSK9 concentrations in control $(\mathrm{CON})$, pair-fed (PF) and chronic renal failure (CRF) rats: a Serum total cholesterol (TCh) concentration (mean $\pm \mathrm{SD}$; $n=10$ per group); $\mathbf{b}$ Serum LDL-cholesterol (LDL-Ch) concentration (mean $\pm \mathrm{SD}$; $n=10$ per group); c Relative liver PCSK9 mRNA level expressed as arbitrary unit (a. u.) The mean $\pm \mathrm{SD}$ was obtained from 10 rats per group; d The representative Western blot analysis of liver PCSK9 protein level. Actin was used as a standard; e Serum PCSK9 concentration determined by ELISA (mean $\pm \mathrm{SD} ; n=10$ per group); $\mathbf{f}$ The relationship between serum PCSK9 concentration and liver PCSK9 mRNA level (based on data presented in a and c). Statistics: $* p<0.001$
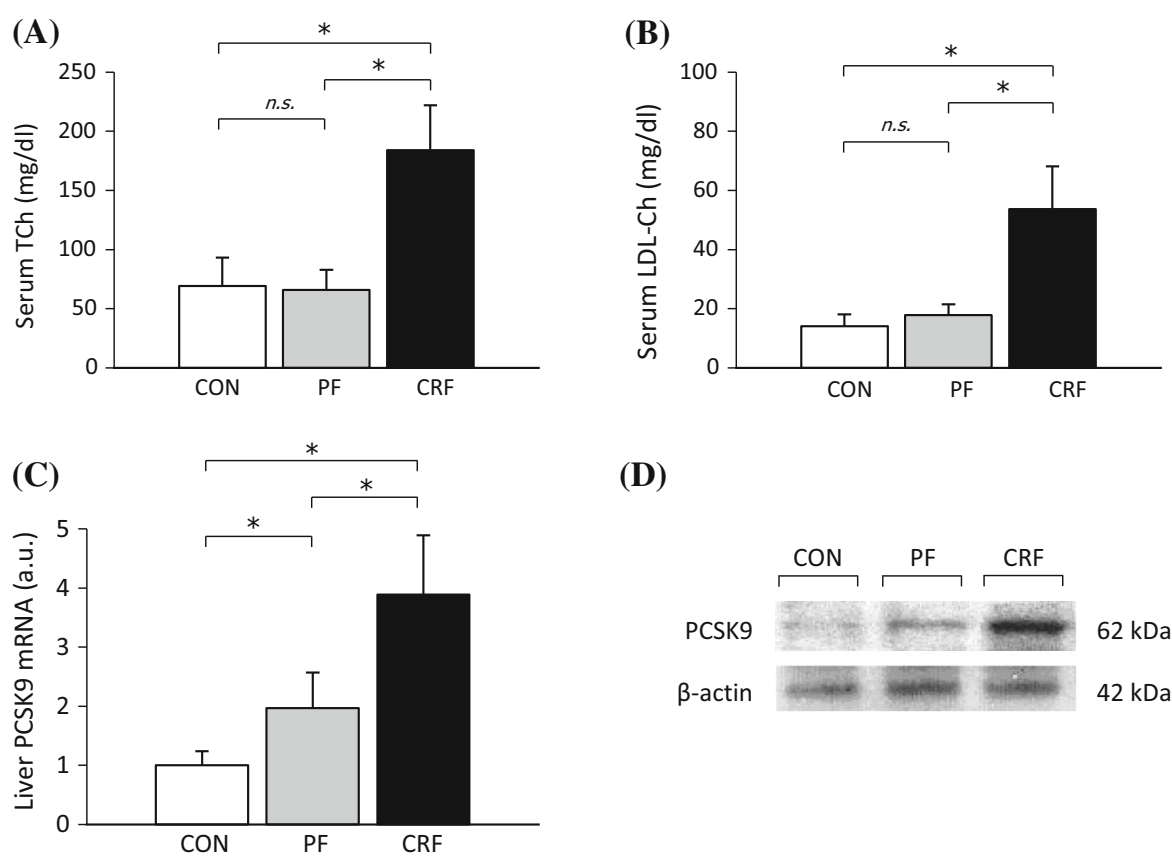

(D)

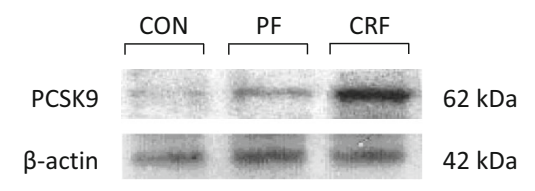

(F)

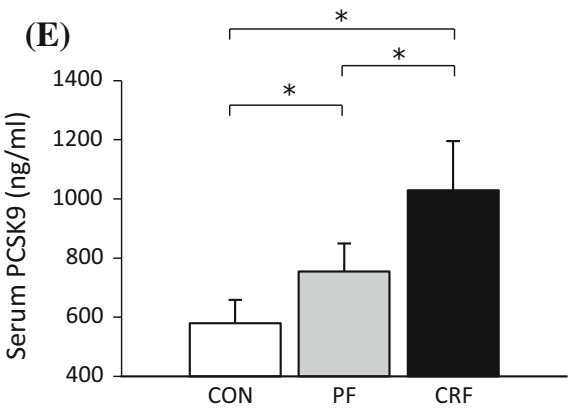

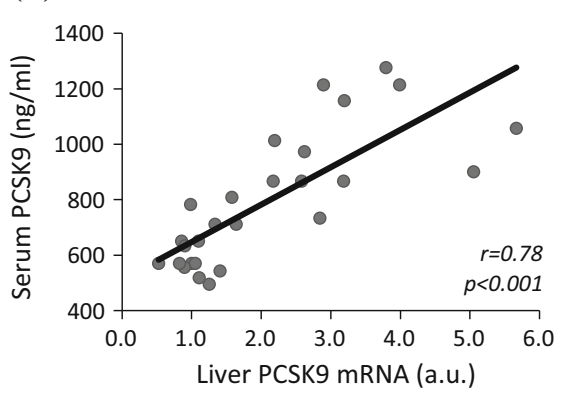

PCSK9 mRNA was found in CRF rats (approximately 3.5fold greater that in $\mathrm{CON}$ and approximately twofold greater as compared to PF). The differences in liver PCSK9 mRNA levels in CON, PF, and CRF rats were paralleled by differences in liver PCSK9 protein amounts determined by Western blot analysis (Fig. 1d) and serum PCSK9 concentrations determined by ELISA (Fig. 1e). Moreover, we found positive correlations between serum PCSK9 concentration and liver PCSK9 both mRNA level (Fig. 1f) and PCSK9 protein amount $(r=0.93 ; p<0.001)$ (data not shown). Furthermore, we observed positive correlations between serum creatinine concentration and liver PCSK9 mRNA (Fig. 2a) and circulating PCSK9 (Fig. 2b) levels. This suggests that Pcsk9 gene expression and serum PCSK9 concentration are closely related to renal failure.

Serum TCh and LDL-Ch concentrations are positively correlated with a circulating PCSK9 level (Fig. 2c, d). Moreover, the increased liver Pcsk 9 gene expression and subsequently the increased circulating PCSK9 level were accompanied by a decreased liver LDL-R protein amount in CRF rats (Fig. 3). Inverse associations between a liver LDL-R protein amount and serum TCh $(r=-0.75$; $p<0.001)$ or LDL-Ch $(r=-0.81 ; p<0.001)$ concentrations were also found (data not shown). Presumably, this partially contributes to hypercholesterolemia observed in rat model of CRF.

Since SREBP-2 (product of Srebf-2) is an important regulator of $P \operatorname{csk} 9$ gene expression [21, 22], we determined liver Srebf-2 gene expression and its association with both liver PCSK9 mRNA and serum PCSK9 levels in CON, PF, and CRF rats. Experimental CRF was associated with a significant increase of liver SREBF-2 mRNA level as compared to CON and PF (Fig. 4a). The differences in liver SREBF-2 mRNA levels were paralleled by differences in SREBP-2 protein amounts determined by Western blot analysis (Fig. 4b). The pattern of changes in liver SREBF-2 mRNA levels of CON, PF, and CRF rats resembles the changes in liver PCSK9 mRNA and protein levels (Fig. 1c, d). Finally, strong positive correlations between liver SREBF-2 mRNA and protein mature form 
Fig. 2 The relationships between: a serum creatinine concentration and liver PCSK9 mRNA levels expressed as arbitrary unit (a. u.); b serum creatinine concentration and circulating PCSK9 levels (determined by ELISA); c serum concentrations of PCSK9 (determined by ELISA) and serum total cholesterol (TCh) concentrations); d serum concentrations of PCSK9 (determined by ELISA) and LDL-cholesterol (LDL-Ch) concentrations
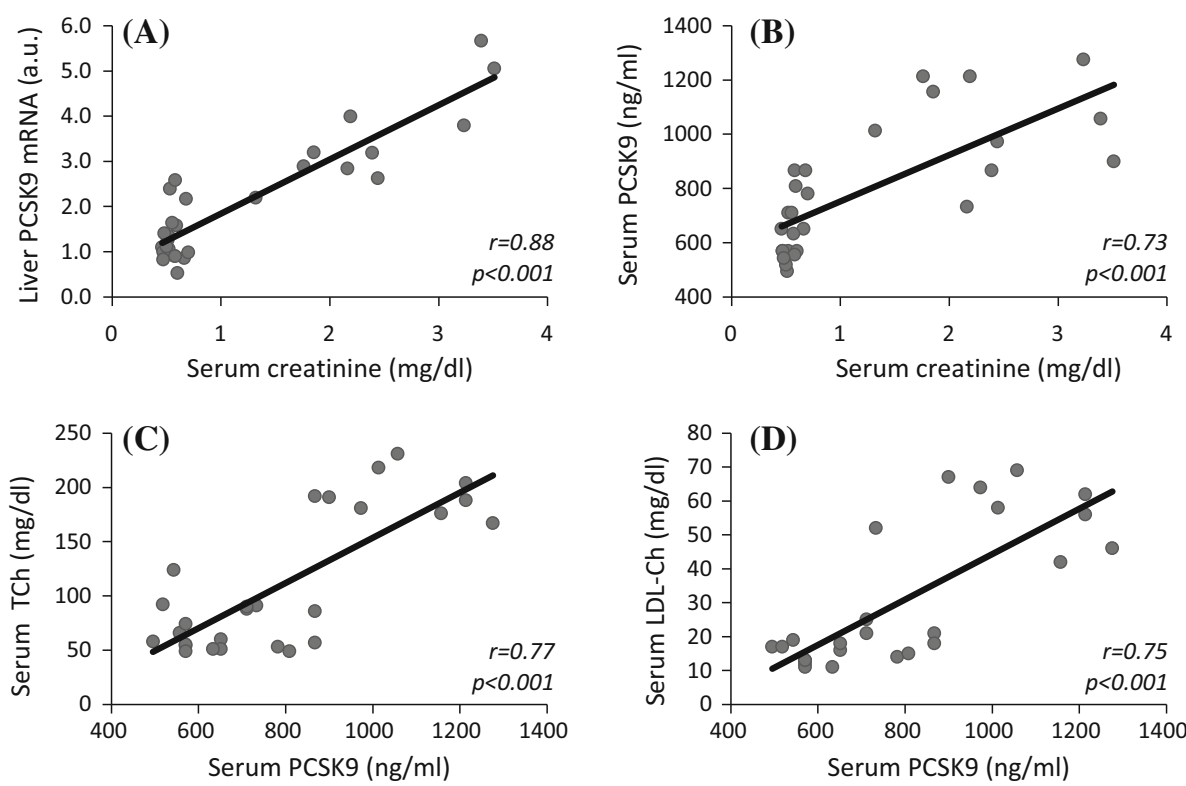

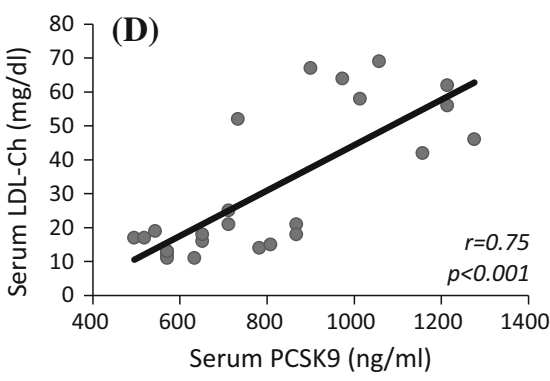

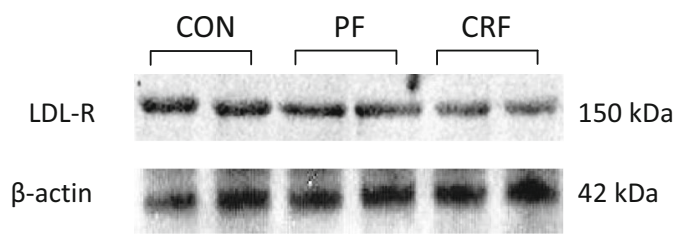

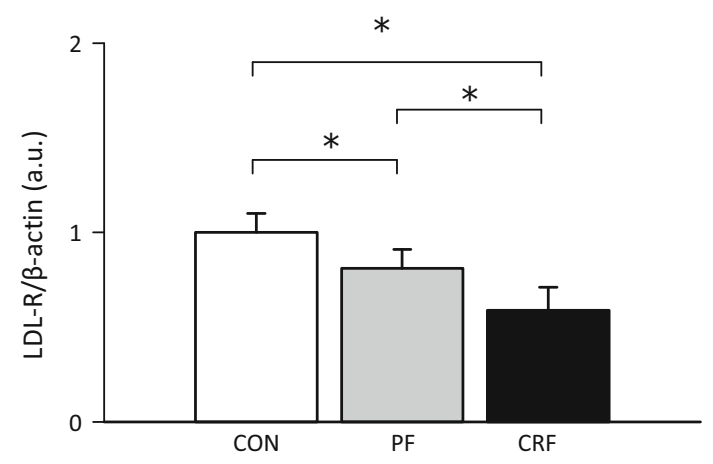

Fig. 3 The representative Western blot analysis (upper panel) and group data (lower panel; the mean $\pm \mathrm{SD}$ was obtained from 10 rats per group) depicting the liver LDL-receptor (LDL-R) protein level in control (CON) pair-fed (PF) and chronic renal failure (CRF) rats. Actin was used as a standard. Statistics: $* p<0.001$

levels versus liver PCSK9 mRNA and serum PCSK9 concentrations were also observed (Fig. 5a-d).

\section{Discussion}

It is generally accepted that PCSK9 is primarily synthesized in liver [27] and that its circulating form is mainly cleared by the liver through LDL-R [6, 7, 9]. It means that
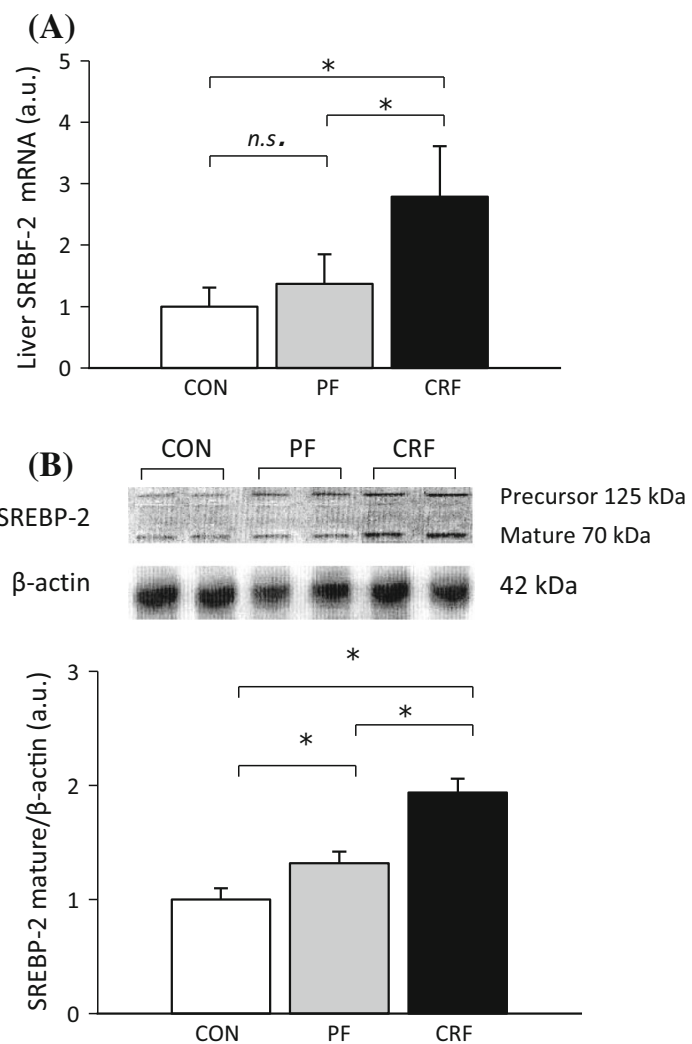

Fig. 4 Liver Srebf-2 gene expression in control (CON), pair-fed (PF) and chronic renal failure (CRF) rats: a Relative liver SREBF-2 mRNA level presented as arbitrary unit (a. u.; mean $\pm \mathrm{SD} ; n=10$ per group). b The representative Western blot analysis (upper panel) and group data (lower panel; the mean $\pm \mathrm{SD}$ was obtained from 10 rats per group) depicting the liver precursor and mature SREBB-2 protein level. Actin was used as a standard. Statistics: $* p<0.001$ 
Fig. 5 The relationships between: a liver SREBF-2 mRNA and liver PCSK9 mRNA levels expressed in arbitrary unit (a. u.); b liver SREBF-2 mRNA level expressed in arbitrary unit (a. u.) and serum PCSK9 concentration (determined by ELISA); c liver SREBP-2 mature form level expressed in arbitrary unit (a. u.) and liver PCSK9 mRNA level expressed in arbitrary unit (a. u.); d liver SREBP-2 mature form level expressed in arbitrary unit (a. u.) and serum PCSK9

concentration determined by ELISA
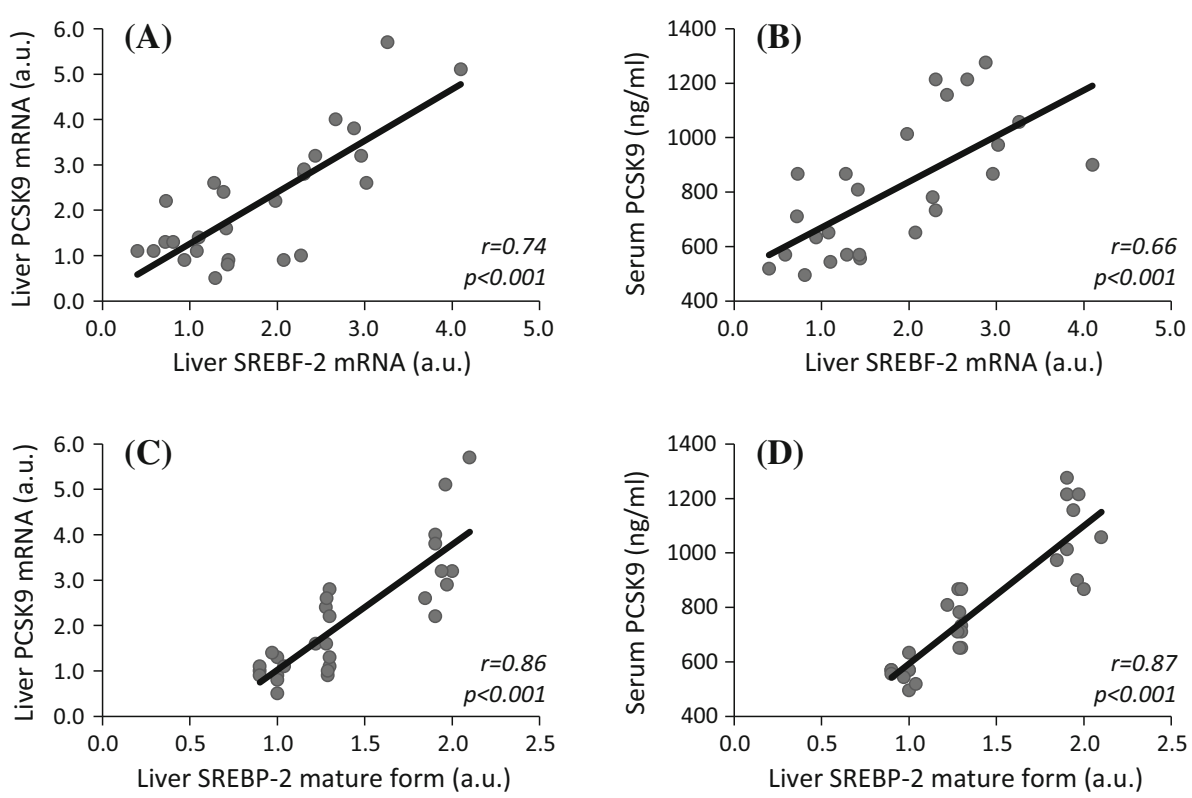

a liver is the main organ regulating serum PCSK9 concentration. Recently, we postulated that PCSK9 overproduction could partially contribute to its elevated serum concentration found in patients with CKD [20]. In the present study, to gain a better insight into the role of PCSK9 in a development of CRF-related hypercholesterolemia, we examined liver Pcsk 9 gene expression in rat model of this disease. Moreover, we explored a possible link between liver Pcsk9, Srebf-2 (SREBP-2 as a positive regulator of $P \operatorname{csk} 9$ gene expression) genes expression and hypercholesterolemia observed in rats with CRF. The data presented herein prove for the first time that an experimental CRF is associated with a coordinated up-regulation of both liver Pcsk9 and liver Srebf-2. Thus, it is likely that up-regulation of Srebf-2 gene causes an elevation of circulating PCSK9 level. The increase of all liver PCSK9 mRNA and protein levels suggests that in experimental CRF, circulating PCSK9 concentration is regulated at the gene transcription level, similarly as in other pathophysiological conditions [21, 22]. As expected, the increased circulating PCSK9 concentration is associated with a lower level of liver LDL-R. The data suggest that the loss of LDL-R impairs LDL-Ch clearance, and consequently leads to serum TCh and LDL-Ch accumulation. Our data also confirm that CRF is associated with an up-regulation of liver Srebf-2 gene expression.

It is obvious that we cannot exclude other mechanisms contributing to PCSK9 up-regulation found in our experimental model of CRF. As it was recently presented, upregulation of PCSK9 gene expression could also be mediated by protein urinary loss in subjects with nephrotic syndrome [19] or by protein peritoneal loss commonly observed in peritoneal dialysis patients [18]. Data from our studies indicate lower plasma total protein (59.2 $\pm 7.1 \mathrm{vs.}$ $66.4 \pm 11.1 \mathrm{~g} / \mathrm{l} ; p<0.01)$ and albumin $(3.4 \pm 0.2$ vs. $4.0 \pm 0.4 \mathrm{~g} / \mathrm{dl} ; p<0.001$ ) (not shown) levels in CRF rats as compared with $\mathrm{CON}$ ones. This is presumably due to rat model of CRF-related proteinuria, which could mediate elevation of serum PCSK9 level [28]. However, the strong positive correlation found between liver PCSK9 mRNA level and serum creatinine concentration (Fig. 2a) suggests that CRF also partially contributes to PCSK9 up-regulation. This assumption is also supported by other results, i.e., positive correlation between serum creatinine and PCSK9 concentrations (Fig. 2b) and positive correlation between liver SREBP-2 mRNA and PCSK9 mRNA levels (Fig. 5a).

All the presented data allow us to recognize that PCSK9 plays an important role in experimental CRF-related lipid metabolism disorders. Due to obvious limitations, most of these studies cannot be performed in patients with this disease. However, it should be at least considered how these findings are related to human CKD. Based on the data reported recently [20], we can only hypothesize that a similar up-regulation of liver hepatic transcriptional factor and subsequent stimulation of $P \operatorname{csk} 9$ gene expression could be found in CKD patients.

In conclusion, the results presented in this paper indicate that experimental model of CRF is associated with an increased liver Pcsk 9 gene expression, an enhanced circulating PCSK9 concentration and in consequence, a decreased liver LDL-R level. All these contribute to CRFrelated hypercholesterolemia. Coordinated up-regulation of Srebf-2 and Pcsk9 genes expression suggests that SREBP-2 
may play a crucial role in regulation of Pcsk 9 gene expression and consequently contribute to hypercholesterolemia observed in subjects with CKD.

Acknowledgments The study was supported by grants from the Polish Committee for Scientific Research via Medical University of Gdansk (ST-4 and ST-41).

\section{Compliance with ethical standards}

Disclosure All authors declare that they have no conflict of interest.

Open Access This article is distributed under the terms of the Creative Commons Attribution 4.0 International License (http://crea tivecommons.org/licenses/by/4.0/), which permits unrestricted use, distribution, and reproduction in any medium, provided you give appropriate credit to the original author(s) and the source, provide a link to the Creative Commons license, and indicate if changes were made.

\section{References}

1. Oda H, Keane WF (1998) Lipid abnormalities in end stage renal disease. Nephrol Dial Transplant 13(Suppl 1):45-49

2. Sun L, Halaihel N, Zhang W, Rogers T, Levi M (2002) Role of sterol regulatory element-binding protein 1 in regulation of renal lipid metabolism and glomerulosclerosis in diabetes mellitus. J Biol Chem 277:18919-18927

3. Szolkiewicz M, Sucajtys E, Chmielewski M, Wolyniec W, Rutkowski P, Boguslawski W, Swierczynski J, Rutkowski B (2002) Increased rate of cholesterologenesis-a possible cause of hypercholesterolemia in experimental chronic renal failure in rats. Horm Metab Res 34:234-237

4. Chmielewski M, Sucajtys E, Swierczynski J, Rutkowski B, Bogusławski W (2003) Contribution of increased Hmg-CoA reductase gene expression to hypercholesterolemia in experimental chronic renal failure. Mol Cell Biochem 246:187-191

5. Chmielewski M, Sucajtys-Szulc E, Kossowska E, Swierczynski J, Rutkowski B, Boguslawski W (2007) Increased gene expression of liver Srebp-2 in experimental chronic renal failure. Atherosclerosis 191:326-332

6. Lopez D (2008) PCSK9: an enigmatic protease. Biochim Biophys Acta 1781:184-191

7. Lambert G, Charlton F, Rye KA, Piper DE (2009) Molecular basis of PCSK9 function. Atherosclerosis 203:1-7

8. Lambert G, Sjouke B, Choque B, Kastelein J, Hovingh GK (2012) The PCSK9 decade. J Lipid Res 53:2515-2524

9. Stein EA, Swergold GDP (2013) Potential of proprotein convertase subtilisin/kexin type 9 based therapeutics. Curr Atheroscler Rep 15:310. doi:10.1007/s11883-013-0310-3

10. Seidah NG, Benjannet S, Wickham L, Marcinkiewicz J, Jasmin SB, Stifani S, Basak A, Prat A, Chretien M (2003) The secretory proprotein convertase neural apoptosis-regulated convertase 1 (NARC-1): liver regeneration and neuronal differentiation. Proc Natl Acad Sci USA 100:928-933

11. Seidah NG, Prat A (2007) The proprotein convertases are potential targets in the treatment of dyslipidemia. J Mol Med (Berl) 85:685-696

12. Urban D, Poss J, Bohm M, Laufs U (2013) Targeting the proprotein convertase subtilisin/kexin type 9 for the treatment of dyslipidemia and atherosclerosis. J Am Coll Cardiol 62:1401-1408

13. Rashid S, Curtis DE, Garuti R, Anderson NN, Bashmakov Y, Ho YK, Hammer RE, Moon YA, Horton JD (2005) Decreased plasma cholesterol and hypersensitivity to statins in mice lacking PCSK9. Proc Natl Acad Sci USA 102:5374-5379

14. Zaid A, Roubtsova A, Essalmani R, Marcinkiewicz J, Chamberland A, Hamelin J, Tremblay M, Jacques H, Jin W, Davignon J, Seidah NG, Prat A (2008) Proprotein convertase subtilisin/ kexin type 9 (PCSK9): hepatocyte-specific low-density lipoprotein receptor degradation and critical role in mouse liver regeneration. Hepatology 48:646-654

15. Alborn WE, Cao G, Careskey HE, Qian YW, Qian YW, Subramaniam DR, Davies J, Conner EM, Konrad RJ (2007) Serum proprotein convertase subtilisin kexin type 9 is correlated directly with serum LDL cholesterol. Clin Chem 53:1814-1819

16. Lee ChJ, LeeY-ho Parc SW, Kim KJ, Park S, Youn J-Ch, LeeS-H Kang S-M, Jang Y (2013) Association of serum proprotein convertase subtilisin/kexin type 9 with carotid intima media thickness in hypertensive subjects. Metabolism 62:845-850

17. Blom DJ, Hala T, Bolognese M, Lillestol MJ, Toth PD, Burgess L, Ceska R, Roth E, Koren MJ, Ballantyne CM, Monsalvo ML, Tsirtsonis K, Kim JB, Scott R, Wasserman SM, Stein EA (2014) DESCARTES investigators: a 52-week placebo-controlled trial of evolocumab in hyperlipidemia. N Engl J Med 370:1809-1819

18. Jin K, Park BS, Kim YW, Vaziri ND (2014) Plasma PCSK9 in nephrotic syndrome and in peritoneal dialysis: a cross-sectional study. Am J Kidney Dis 63:584-589

19. Liu S, Vaziri ND (2014) Role of PCSK9 and IDOL in the pathogenesis of acquired LDL receptor deficiency and hypercholesterolemia in nephrotic syndrome. Nephrol Dial Transplant 29:538-543

20. Konarzewski M, Szolkiewicz M, Sucajtys-Szulc E, Blaszak J, Lizakowski S, Swierczynski J, Rutkowski B (2014) Elevated circulating PCSK-9 concentration in renal failure patients is corrected by renal replacement therapy. Am J Nephrol 40:157-163

21. Jeong HJ, Lee HS, Kim KS, Kim YK, Yoon D, Park SW (2008) Sterol-dependent regulation of proprotein convertase subtilisin/ kexin type 9 expression by sterol-regulatory element binding protein-2. J Lipid Res 49:399-409

22. Costet P, Cariou B, Lambert G, Lalanne F, Lardeux B, Jarnoux AL, Grefhorst A, Staels B, Krempf M (2006) Hepatic PCSK9 expression is regulated by nutritional status via insulin and sterol regulatory element-binding protein 1c. J Biol Chem 281:6211-6218

23. Swierczynski J, Korczynska J, Szolkiewicz M, Karbowska J, Kochan Z, Nieweglowski T, Kusiak E, Rutkowski B (2001) Low leptin mRNA level in adipose tissue and normoleptinemia in experimental chronic renal failure. Exp Nephrol 9:54-59

24. Turyn J, Stojek M, Swierczynski J (2010) Up-regulation of stearol-CoA desaturase 1 and elongase 6 genes expression in rat lipogenic tissues by chronic food restriction and chronic food restriction/refeeding. Mol Cell Biochem 345:181-188

25. Chomczynski P, Sacchi N (1987) Single-step method of RNA isolation by acid guanidinium thiocyanate-phenol-chloroform extraction. Anal Biochem 162:156-159

26. Winer J, Jung CK, Shackel I, Williams PM (1999) Development and validation of real-time quantitative reverse transcriptasepolymerase chain reaction for monitoring gene expression in cardiac myocytes in vitro. Anal Biochem 270:41-49

27. Roubtsova A, Munkonda MN, Awan Z, Marcinkiewicz J, Chamberland A, Lazure C, Cianflone K, Seidah NG, Prat A (2011) Circulating proprotein convertase subtilisin/kexin 9 (PCSK9) regulates VLDLR protein and triglyceride accumulation in visceral adipose tissue. Arterioscler Thromb Vasc Biol 31:785-791

28. Kwakernaak AJ, Lambert G, Slagman MC, Waanders F, Laverman GD, Petrides F, Dikkeschei BD, Navis G, Dullaart RP (2013) Proprotein convertase subtilisin-kexin type 9 is elevated in proteinuric subjects: relationship with lipoprotein response to antiproteinuric treatment. Atherosclerosis. 226:459-465 